\title{
Survival impact of adherence to tyrosine kinase inhibitor in chronic myeloid leukemia
}

\author{
Yundeok Kim ${ }^{1}$ Tae-Hwa Go², Jaeyeon Jang ${ }^{1}$, Jii Bum Lee ${ }^{1}$, Seung Taek Lim ${ }^{1}$ Kwang Yong Shim , \\ Jong In Lee ${ }^{1}$, and Jee Hyun Kong ${ }^{1,3}$
}

\begin{abstract}
${ }^{1}$ Division of Hematology-Oncology, Department of Internal Medicine, Wonju Severance Christian Hospital, Yonsei University Wonju College of Medicine, Wonju; ${ }^{2}$ Department of Biostatics, Yonsei University Wonju College of Medicine, Wonju; ${ }^{3}$ Cancer of Evidence Based Medicine, Institute of Convergence Science, Yonsei University, Seoul, Korea
\end{abstract}

Received: March 25, 2021

Revised : June 21, 2021

Accepted: July 21, 2021

\section{Correspondence to}

Jee Hyun Kong, M.D.

Division of Hematology-

Oncology, Department of

Internal Medicine, Wonju

Severance Christian Hospital,

Yonsei University Wonju College of Medicine, 20 Ilsan-ro, Wonju

26426, Korea

Tel: $+82-33-741-0511$

Fax: +82-33-741-3308

E-mail: kkongg@yonsei.ac.kr

https:/orcid.org/0000-0001-

$7833-8480$
Background/Aims: Adherence to tyrosine kinase inhibitors (TKIs) has become a critical aspect of care in chronic myeloid leukemia (CML). We aimed to examine the association of TKI adherence with overall survival (OS) outcomes in Korean patients diagnosed with CML and treated with TKIs using data from the National Health Information Database.

Methods: This study included 2,870 CML patients diagnosed between 2005 and 2013. Drug adherence was evaluated according to the medication possession ratio (MPR) and classified as high adherence (i.e., MPR $\geq 0.95$ [upper 50\%]), moderate adherence (i.e., MPR $\geq 0.68$ and $<0.95$ [middle 25\%]), and low adherence (i.e., MPR $<0.68$ [lower 25\%]).

Results: The median MPR was 0.95 (range, o to 4.67 ). Male sex $(p=0.003$ ), age $<70$ years $(p<0.001)$, high income $(\geq 30 \%, p<0.001)$, and maintaining frontline TKI $(<$ $0.001)$ were associated with better adherence. Adherence to dasatinib was the lowest (vs. imatinib or nilotinib, $p<0.001$ ). Compared with high MPR patients, those with moderate MPR (hazard ratio [HR], 4.90; 95\% confidence interval [CI], 3.87 to 6.19; $p<0.001$ ) and low MPR (HR, 11.6; 95\% CI, 9.35 to 14.42; $p<0.001)$ had poorer OS.

Conclusions: Adherence to TKI treatment is an important factor predicting survival outcomes in Korean CML patients. Male sex, age $<70$ years, high income, and maintaining frontline TKI are associated with high adherence to TKI. Thus, those without these characteristics should be closely monitored for treatment adherence.

Keywords: Chronic myeloid leukemia; Tyrosine kinase inhibitor; Medication possession ratio; Adherence

\section{INTRODUCTION}

Chronic myeloid leukemia (CML) is a myeloproliferative neoplasm that occurs in 1 to 2 per 100,000 adults. The key to CML pathogenesis is the fusion of the Abelson murine leukemia ( $A B L 1)$ gene on chromosome 9 with the breakpoint cluster region (BCR) gene on chromosome 22. This leads to the expression of an oncoprotein termed BCR-ABL [1]. In this context, the BCR-ABL1 fusion gene is an active tyrosine kinase that advances tumor growth and replication through downstream signaling pathways such as MYC and signal transducer and activator of transcription (STAT) $[2,3]$.

Imatinib and other tyrosine kinase inhibitors (TKIs) have markedly improved the treatment of CML [4]. Several studies have reported that TKIs improve the survival of chronic phase CML patients similar to that of the general population, thereby increasing the 10-year 
survival rate from approximately $20 \%$ to $80 \%-90 \%[4,5]$. Accordingly, studies have assessed whether TKIs can be safely discontinued in patients who have achieved longterm deep molecular response (DMR).

Current European LeukemiaNet (ELN) [6] guidelines stipulate that treatment can be successfully stopped if both treatment duration and DMR are adequate to make treatment-free remission feasible for a potential cure. However, among patients who become candidates for the termination of TKI therapy after achieving adequate DMR, the incidence of molecular cure remains relatively low at approximately $20 \%-25 \%$ for imatinib and $30 \%-45 \%$ for second-generation TKIs $[5,7]$.

Adherence to TKI has also become a critical aspect of care in CML [8]. As such, there have been studies on survival outcomes according to patient adherence levels [9]. Strict adherence increases the probability of clinical success [10]. Low adherence to imatinib was reported to be associated with a lower rate of cytogenetic and molecular responses in CML and consequently a higher rate of progression and resistance $[8,11]$.

The National Health Insurance Service (NHIS) in Korea is the compulsory health insurance scheme covering the whole Korean population [12]. The National Health Information Database (NHID), formed by NHIS, has been developed for public health and medical research purposes and provides personal information, demographics, and medical treatment data of Korean citizens [13]. NHID stores the claims data of approximately 46 million patients per year, which accounts for around $90 \%$ of the total population in Korea. It also includes claims from 80,000 healthcare service providers as of 2011.

This study aimed to examine the association of TKI adherence with overall survival (OS) outcomes and clinical parameters influencing survival outcomes in CML patients treated with TKIs. Toward this goal, we investigated practice trends and clinical outcomes in a large community-based integrated health plan in South Korea, i.e., the NHID database.

\section{METHODS}

\section{Study design and patients}

This was a retrospective study of 3,916 adult CML pa- tients with a confirmed diagnosis of Philadelphia-positive chromosome and with a prescription for TKIs more than once between 2005 and 2013. Patients treated with TKIs used in clinical trials or those who were not covered by NHIS (e.g., ponatinib or bosutinib) were unavailable. After excluding 1,046 patients owing to incomplete clinical data $(\mathrm{n}=382)$, previous treatment with interferon- $\alpha$ before TKI administration $(n=14)$, prescription with TKI only once $(n=58)$, and TKI at 3 months after diagnosis $(n=268)$. We wanted to include chronic phase patients, and the International Classification of Diseases for Oncology, 3rd edition morphology code (ICD-O-3 921) does not differentiate CML phases, high-dose TKIs (i.e., imatinib, > $400 \mathrm{mg} /$ day; dasatinib, $100 \mathrm{mg} /$ day; nilotinib, $600 \mathrm{mg} /$ day; and radotinib, $600 \mathrm{mg} /$ day) which were usually prescribed at CML in accelerating or blast phase ( $\mathrm{n}=323)$, and cytotoxic chemotherapy within 1 year after diagnosis $(n=1)$ were excluded (Fig. 1).

The patients were identified in the database according to the ICD-O-3 codes for CML (ICD-O-3 92.1) [14]. We extracted data on age, sex, medical insurance premium, prescription records (drug code, days prescribed, daily dosage), date of hematopoietic stem cell transplantation (HSCT), and date of death. Prescription data on TKIs (imatinib, dasatinib, nilotinib, and radotinib) covered by NHIS between 2005 and 2013 were gathered to ana-

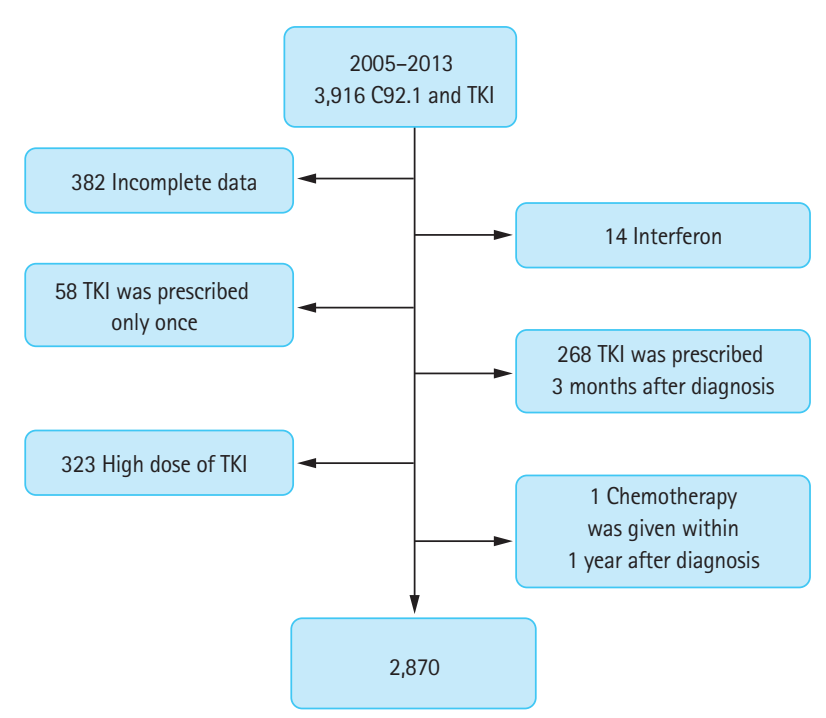

Figure 1. Consort diagram. C92.1, codes for CML, International Classification of Diseases for Oncology, 3rd edition (ICD-O-3); TKI, tyrosine kinase inhibitor. 
lyze the treatment pattern for CML. For survival analysis, passive follow-up was performed until December 31, 2016, using the mortality database from statistics Korea.

The medical insurance premium is imposed in proportion to income, and thus, it was considered an indirect marker for economic status. High and low incomes were defined as paying the upper 50\% and lower 50\% of medical insurance premium. Co-medication was defined as the prescription of a second medication within 1 year to alleviate the side effects of another medication. Adherence was measured according to the medication possession ratio (MPR), which was calculated as, the number of days the medication was supplied for, divided by the number of days between the first refill and last follow-up [15]. MPRs of $\geq 0.95$ (upper 50\%), $\geq 0.68$ and $<$ 0.95 (middle 25\%), and < 0.68 (lower 25\%) were defined to indicate high, moderate, and low adherence, respectively.

This study was approved by the Wonju Severance Christian Hospital Institutional Review Board (CR317116). The need for informed consent was waived owing to the retrospective nature of the study.

\section{Statistical analysis}

We defined "event" as a change of TKI, receiving chemotherapy, HSCT, or death. Event-free survival (EFS) was calculated from the first day of diagnosis to the occurrence of the events or last follow-up. It was estimated only in patients who were treated with frontline imatinib. OS was calculated from the first day of diagnosis to the date of death or last follow-up. Continuous and categorical variables were compared using Student's $t$ test (Mann-Whitney test) or analysis of variance and using the chi-square test (Fisher exact test), respectively. Multivariate analysis was performed using Cox proportional hazards models. Variables included in the univariate and multivariate analyses on EFS and OS were sex, medical insurance premium (high vs. low), age ( $\leq 50$ years vs. $>50$ years), and MPR (upper vs. middle; upper vs. lower).

Two-sided 95\% confidence intervals (CIs) were calculated where appropriate. All statistical analyses were performed using SAS for Windows version 9.2 (SAS Institute, Cary, NC, USA). A $p<0.05$ was considered statistically significant.

\section{RESULTS}

\section{Patient characteristics}

The median patient age was 51 years (range, 15 to 92), and 1,747 and 1,123 patients were male and female, respectively. The patient characteristics are summarized in Table 1. Approximately two-thirds of patients $(\mathrm{n}=$ 1,810, 63\%) had a high income. Imatinib was the most frequently prescribed TKI ( $\mathrm{n}=2,476,86.3 \%$ ), followed by dasatinib ( $\mathrm{n}=809,28.2 \%$ ), nilotinib ( $\mathrm{n}=711,24.8 \%$ ), and radotinib ( $\mathrm{n}=132,4.6 \%)$. Approximately one-third of the patients $(\mathrm{n}=982,34.3 \%)$ discontinued first-line TKI. Treatment was switched to the second, third, and fourth-line TKI in 734 (25.6\%), 220 (7.7\%), and 28 (1.0\%) patients, respectively. Imatinib was selected as frontline TKI in 2,417 (84.2\%) patients. Cytotoxic chemotherapy

Table 1. Patient characteristics $(n=2,870)$

\begin{tabular}{|c|c|}
\hline Variable & Value \\
\hline Male sex & $1,747(60.9)$ \\
\hline Age, yr & $51(15-92)$ \\
\hline \multicolumn{2}{|l|}{ TKI } \\
\hline Imatinib & $2,476(86.3)$ \\
\hline Dasatinib & $809(28.2)$ \\
\hline Nilotinib & $711(24.8)$ \\
\hline Radotinib & $132(4 \cdot 6)$ \\
\hline \multicolumn{2}{|l|}{ Number of TKIs } \\
\hline 1 & $1,888(65.8)$ \\
\hline 2 & $734(25 \cdot 6)$ \\
\hline 3 & $220(7.7)$ \\
\hline 4 & $28(1.0)$ \\
\hline \multicolumn{2}{|l|}{ Frontline TKI } \\
\hline Imatinib & $2,417(84.2)$ \\
\hline Others & $453(15.8)$ \\
\hline Chemotherapy & $164(5.7)$ \\
\hline \multicolumn{2}{|c|}{ Hematopoietic stem cell transplantation } \\
\hline Allogeneic & $11(0.4)$ \\
\hline Autologous & $1(0.0)$ \\
\hline \multicolumn{2}{|l|}{ Income } \\
\hline High & $1,810(63.1)$ \\
\hline Low & $1,060(36.9)$ \\
\hline Alive & $2,400(83.6)$ \\
\hline
\end{tabular}

Values are presented as number (\%) or median (range). TKI, tyrosine kinase inhibitor. 


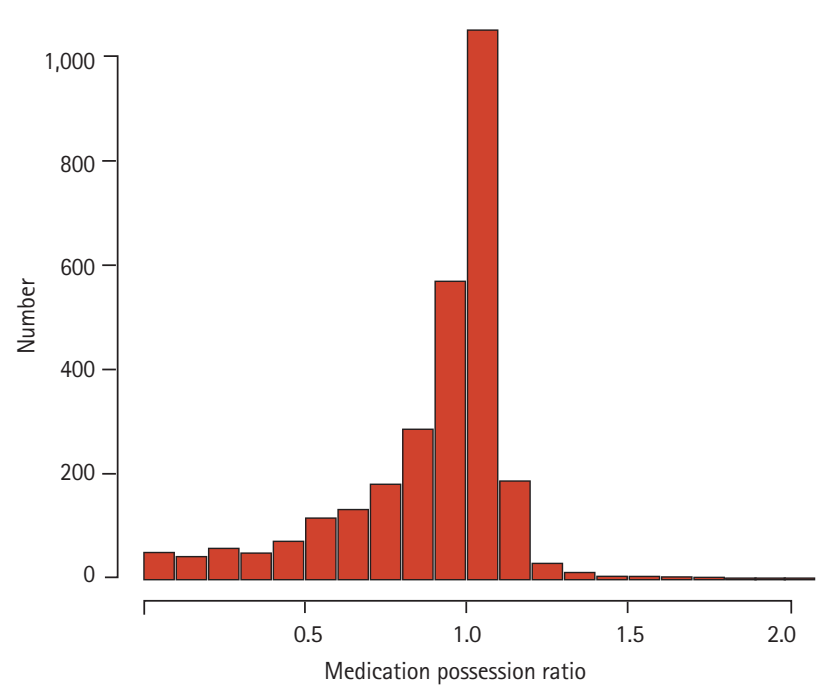

Figure 2. Histogram of medication of possession ratio of tyrosine kinase inhibitors in chronic myeloid leukemia.

was administered to 164 patients (5.7\%), and 12 (0.4\%) patients underwent HSCT.

\section{Influencing factors of TKI adherence}

Adherence to TKIs as reflected by MPR is shown in Fig. 2. The median MPR was 0.99 (range, 0.00 to 2.03), and 1,292 (62.1\%) patients had an MPR $\geq 1$.o. The median MPRs of the high $(n=1,414)$, moderate $(n=746)$, and low $(\mathrm{n}=710)$ adherence groups were 1.05 (range, 0.99 to 2.03), 0.93 (range, 0.80 to 0.99 ), and 0.57 (range, o to 0.79 ), respectively. The median MPR according to patient characteristics is summarized in Table 2. The median MPR was significantly higher in male individuals (vs. female individuals, $p=0.003$ ), patients paying high insurance premium (vs. those not paying, $p<0.001$ ), and those who maintained frontline TKI (vs. those who did not, $p<$ o.001). The median MPR was the lowest in patients treated with dasatinib (vs. imatinib or nilotinib, $p<0.001$ ). Only 132 (4.6\%) patients were treated with radotinib, and thus, no statistical analysis could be performed between patients treated with radotinib and those treated with other TKIs. With respect to age group, elderly patients $(\geq 70$ years) had the lowest MPR $(p<0.001)$. The number of concomitant medications (o-3 vs. $\geq 4$ ) did not affect adherence to TKIs $(p=0.832)$.
Table 2. Median MPR with respect to patient characteristics

\begin{tabular}{|c|c|c|}
\hline Variable & Median MPR & $p$ value \\
\hline \multicolumn{3}{|l|}{ Sex } \\
\hline Male & $0.99(0.00-2.03)$ & 0.003 \\
\hline Female & $0.98(0.01-1.55)$ & \\
\hline \multicolumn{3}{|l|}{ Age, yr } \\
\hline${ }^{a} 15-29$ & $1.00(0.01-2.03)$ & $\begin{array}{l}<0.001 \\
a, b, c>d\end{array}$ \\
\hline$b_{30}-49$ & $1.01(0.00-1.72)$ & \\
\hline$c_{50}-69$ & $0.99(0.01-1.91)$ & \\
\hline $\mathrm{d} \geq 70$ & $0.90(0.01-1.80)$ & \\
\hline \multicolumn{3}{|l|}{ Income } \\
\hline High & $1.00(0.00-1.91)$ & $<0.001$ \\
\hline Low & $0.97(0.01-2.03)$ & \\
\hline \multicolumn{3}{|l|}{ TKI } \\
\hline${ }^{\mathrm{a}}$ Imatinib & $0.98(0.00-2.03)$ & $\begin{array}{l}<0.001 \\
\quad \mathrm{a}, \mathrm{c}>\mathrm{b}\end{array}$ \\
\hline${ }^{\mathrm{b}}$ Dasatinib & $0.94(0.02-1.55)$ & \\
\hline${ }^{\mathrm{c}}$ Nilotinib & 0.98 (0.06-1.69) & \\
\hline${ }^{\mathrm{d}}$ Radotinib & $0.98(0.22-1.41)$ & \\
\hline \multicolumn{3}{|c|}{ Number of TKIs } \\
\hline $\mathrm{a}_{1}$ & $1.01(0.00-2.03)$ & $\begin{array}{l}<0.001 \\
\mathrm{a}>\mathrm{b}, \mathrm{c}, \mathrm{d}\end{array}$ \\
\hline $\mathrm{b}_{2}$ & $0.91(0.02-1.54)$ & \\
\hline$c_{3}$ & $0.94(0.10-1.24)$ & \\
\hline $\mathrm{d}_{4}$ & $0.84(0.47-1.10)$ & \\
\hline \multicolumn{3}{|c|}{ Concomitant medication } \\
\hline $0-3$ & $0.91(0.68-1.14)$ & 0.832 \\
\hline$>4$ & $0.90(0.70-1.10)$ & \\
\hline \multicolumn{3}{|l|}{ Frontline TKI } \\
\hline Imatinib & $0.98(0.00-2.03)$ & $<0.001$ \\
\hline Others & $1.02(0.06-1.69)$ & \\
\hline
\end{tabular}

Values are presented as median (range).

MPR, medication possession ratio; TKI, tyrosine kinase inhibitor.

\section{Survival outcomes}

Within a median follow-up of 69.0 months (range, o to 147), 2,400 patients (83.6\%) were alive. The 5- and 10-year OS rates were $87.1 \%$ (95\% CI, $84.3 \%$ to $89.4 \%$ ) and $77.3 \%$ (95\% CI, $75.0 \%$ to $79.4 \%$ ), respectively. Although HSCT was not examined as a study endpoint in the survival analysis, 12 patients underwent this procedure.

Patients were classified into the younger or elderly 

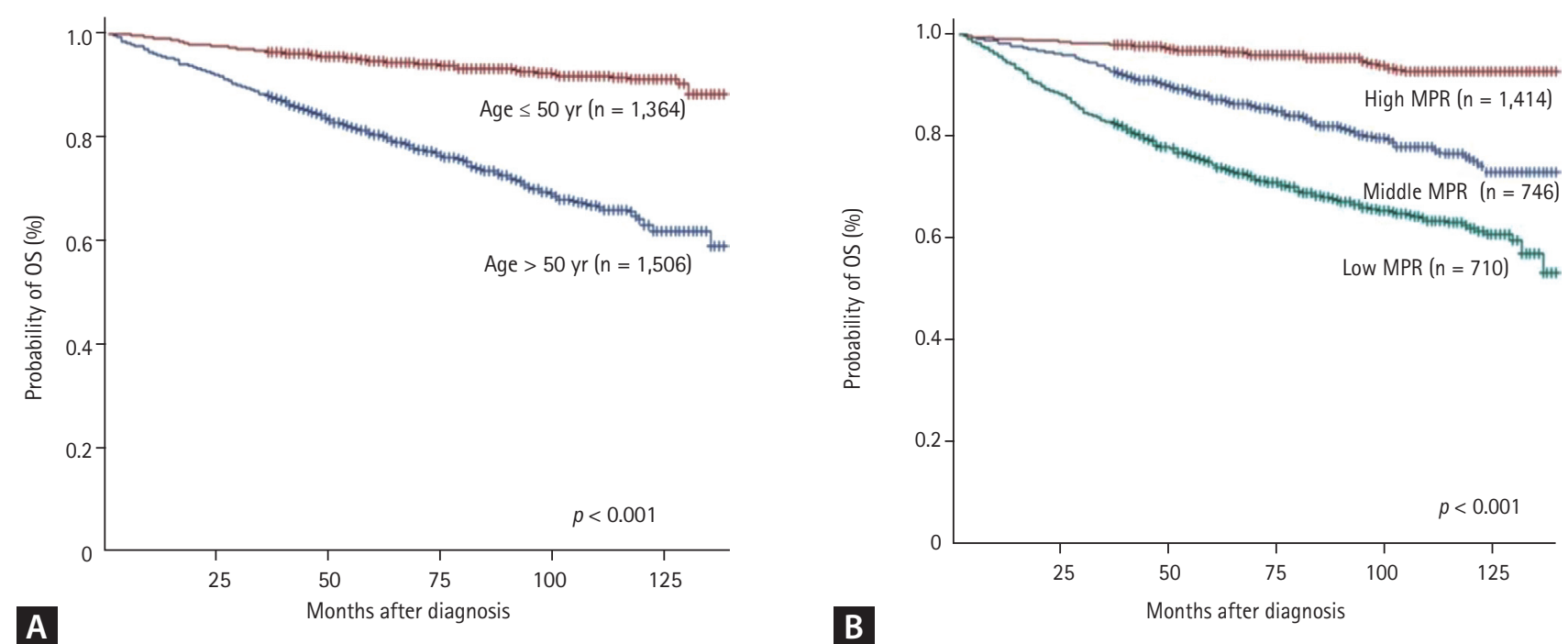

Figure 3. Estimated overall survival (OS). Patients aged $\leq 50$ years (A) and those with high medication possession ratio (MPR) (B) have better OS than those aged $>50$ years or with middle/low MPR $(\log \operatorname{rank} p<0.001$ and $p<0.001$, respectively).

(age $\leq 50$ years vs. $>50$ years) using median age. Elderly at diagnosis $(>50$ years) had inferior OS $(p<0.001)$ (Fig. $3 A)$ and EFS $(p<0.001)$ than younger $(\leq 50$ years). Patients paying high insurance premium showed superior EFS than lower insurance premium $(p=0.015)$.

In the low, moderate, and high adherence group, the 5-year OS rates were $70.2 \%$ (95\% CI, 66.6\% to $73.5 \%$ ), $85.2 \%$ (95\% CI, $82.3 \%$ to $87.6 \%$ ) and $97.2 \%$ (95\% CI, $96.1 \%$ to $98.0 \%$ ), respectively (Fig. $3 \mathrm{~B}$ ). The OS rates were significantly better in the high adherence group than in the middle and low adherence groups (OS in high MPR vs. middle MPR, $p<0.001$; OS in high MPR vs. low MPR, $p$ $<0.001$ ) (Fig. 3B).

\section{Influencing factors of survival in patients treated with imatinib frontline}

We analyzed influencing factors of OS or EFS in patients treated with imatinib as frontline treatment $(n=2,417)$. More than half of patients ( $\mathrm{n}=1,263,52.3 \%)$ were highly adherent to TKI, and 595 (24.6\%) and 559 (23.1\%) patients were revealed middle and low MPR. univariate analysis to identify prognostic factors of OS, age at diagnosis and MPR showed a significant effect on OS $(p<0.001)$ (Table 3). In multivariate analysis, male sex (hazard ratio [HR], 1.43; $95 \%$ CI, 1.18 to $1.75 ; p<0.001$ ), old age (> 50 years) (HR, 3.93; 95\% CI, 3.11 to 4.97; $p<0.001$ ), and middle (HR, 4.62; 95\% CI, 3.30 to 6.47 ; $p<0.001)$ or lower MPR (HR,
9.79; $95 \%$ CI, 7.14 to $13.42 ; p<0.001)$ were predictors of inferior OS (Table 3). In EFS, insurance premium age at diagnosis and MPR was associated EFS significantly in univariate analysis (Table 4). In multivariate analysis, female, high insurance premium, young age, and high MPR had a lower HR of events than male (HR, 1.17; 95\% CI, 1.03 to $1.32 ; p=0.012$ ), low insurance premium (HR, 1.18; $95 \% \mathrm{CI}, 1.04$ to $1.32 ; p=0.008$ ), old age ( $>50$ years) (HR, 1.52; 95\% CI, 1.35 to $1.71 ; p<0.001$ ), and middle MPR (HR, 1.82; 95\% CI, 1.62 to 2.20 ; $p<0.001$ ) or lower MPR (HR, 3.03; 95\% CI, 2.63 to 3.50, $p<0.001$ ) (Table 4).

\section{DISCUSSION}

This national cohort study found that most CML patients treated with TKI were highly adherent to the treatment, and TKI adherence affected survival outcomes. To our best knowledge, this is the first study to demonstrate that TKI adherence affected OS in CML patients. Adherence to TKI was assessed variably in previous reports [16]. Retrospective studies mostly used claims data and pharmacy refill data to assess adherence, with MPR used as the primary indicator [16-18]. The average adherence reported in studies varied from $19 \%$ to $100 \%$, with differences according to the types of measurement methods used [17,19,20]. When an MPR cut-off was used 
Table 3. Univariate and multivariate analyses of influencing factors of overall survival in patients treated with frontline imatinib therapy

\begin{tabular}{|c|c|c|c|c|}
\hline \multirow{2}{*}{ Variable } & \multicolumn{2}{|c|}{ Univariate } & \multicolumn{2}{|c|}{ Multivariate } \\
\hline & $\operatorname{HR}(95 \% \mathrm{CI})$ & $p$ value & $\operatorname{HR}(95 \% \mathrm{CI})$ & $p$ value \\
\hline \multicolumn{5}{|l|}{ Sex } \\
\hline Female & 1.00 (reference) & & 1.00 (reference) & \\
\hline Male & $1.14(0.94-1.39)$ & 0.183 & $1.43(1.18-1.75)$ & $<0.001$ \\
\hline \multicolumn{5}{|c|}{ Medical insurance premium } \\
\hline High & 1.00 (reference) & & 1.00 (reference) & \\
\hline Low & $1.10(0.90-1.33)$ & 0.356 & $1.06(0.87-1.29)$ & 0.560 \\
\hline \multicolumn{5}{|l|}{ Age, yr } \\
\hline$\leq 50$ & 1.00 (reference) & $<0.001$ & 1.00 (reference) & $<0.001$ \\
\hline$>50$ & $4.22(3.34-5.32)$ & & $3.93(3.11-4.97)$ & \\
\hline \multicolumn{5}{|c|}{ Medication possession ratio } \\
\hline Upper $(\geq 0.95)$ & 1.00 (reference) & & 1.00 (reference) & \\
\hline Middle (0.68-0.95) & $5.05(3.61-7.07)$ & $<0.001$ & $4.62(3.30-6.47)$ & $<0.001$ \\
\hline Lower $(<0.68)$ & $10.37(7.57-14.21)$ & $<0.001$ & $9.79(7.14-13.42)$ & $<0.001$ \\
\hline
\end{tabular}

$\mathrm{HR}$, hazard ratio; CI, confidence interval.

Table 4. Univariate and multivariate analyses of influencing factors of event-free survival in patients treated with frontline imatinib therapy

\begin{tabular}{|c|c|c|c|c|}
\hline \multirow{2}{*}{ Variable } & \multicolumn{2}{|c|}{ Univariate } & \multicolumn{2}{|c|}{ Multivariate } \\
\hline & $\mathrm{HR}(95 \% \mathrm{CI})$ & $p$ value & $\operatorname{HR}(95 \% \mathrm{CI})$ & $p$ value \\
\hline \multicolumn{5}{|l|}{ Sex } \\
\hline Female & 1.00 (reference) & & 1.00 (reference) & \\
\hline Male & $1.06(0.94-1.20)$ & 0.331 & $1.17(1.03-1.32)$ & 0.012 \\
\hline \multicolumn{5}{|c|}{ Medical insurance premium } \\
\hline High & 1.00 (reference) & & 1.00 (reference) & \\
\hline Low & $1.20(1.07-1.35)$ & 0.003 & $1.18(1.04-1.32)$ & 0.008 \\
\hline \multicolumn{5}{|l|}{ Age, yr } \\
\hline$\leq 50$ & 1.00 (reference) & $<0.001$ & 1.00 (reference) & $<0.001$ \\
\hline$>50$ & $1.56(1.39-1.76)$ & & $1.52(1.35-1.71)$ & \\
\hline \multicolumn{5}{|c|}{ Medication possession ratio } \\
\hline Upper $(\geq 0.95)$ & 1.00 (reference) & & 1.00 (reference) & \\
\hline Middle (0.68-0.95) & $1.97(1.69-2.29)$ & $<0.001$ & $1.89(1.62-2.20)$ & $<0.001$ \\
\hline Lower $(<0.68)$ & $3.10(2.69-3.57)$ & $<0.001$ & $3.03(2.63-3.50)$ & $<0.001$ \\
\hline
\end{tabular}

HR, hazard ratio; CI, confidence interval.

(usually in the range of $80 \%$ to $90 \%$ ), $44 \%$ to $97 \%$ of patients were classified to be adherent $[17,21]$. The median MPR in this study was 0.99 , and approximately half of the patients $(49.2 \%)$ were classified to have high adher- ence (MPR $\geq 0.95$ ). Despite the full medical insurance coverage including pharmacy benefits, the distribution of MPR still varied widely and was comparable with that in the United States [22]. The mean MPR in this study 
was 0.88 , and approximately two-thirds of the patients had MPR $\geq 0.9$.

With respect to the effect of TKI adherence on patient outcomes, high adherence (i.e., MPR $\geq 0.95$ ) was associated with better EFS and OS. This result was confirmed in the multivariate Cox models, as evidenced by the lower adjusted HRs for event or mortality in patients with high TKI adherence status than those in patients with moderate or low adherence status. Poor adherence may be associated with poor response to treatment [23]. Noens et al. [11] reported that patients with suboptimal response had significantly higher mean percentages of imatinib not taken than did those with an optimal response ( $23 \%$ vs. $7 \%$ using pill count). In addition, a previous study [8] reported that non-adherent patients were less likely to achieve complete cytogenetic responses $(26 \%$ vs. $44 \%, p=0.004)$ at any point. The estimated 5 -year EFS in patients with non-adherence was inferior to that in those with no dose interruptions $(59.8 \%$ vs. $76.6 \%, p=0.011)[9]$.

In the current study, male sex, young age at diagnosis, and high medical insurance premium were associated with survival benefits. Elderly patients ( $\geq 75$ years) do not optimally benefit from TKI treatment, and this could be because of several contributing factors. For instance, these patients experienced more TKI toxicity owing to comorbidities and frailty. In addition, they could have lower treatment adherence and may have even discontinued treatment [24]. In our study, patients aged $>50$ years had inferior OS and EFS to those aged $\leq 50$ years. Patients with high insurance premium had higher adherence than did those with low insurance premium. One study on CML patients in Brazil showed that lowerincome and less economic resources are significantly associated with worse medication adherence [25]. South Korea offers universal access to health care, regardless of the ability to pay, through the NHI and Medical Aid program. The National Health Insurance is financed by compulsory contributions from the insured. The contributions are calculated by considering the insured's income, and thus, patients paying high insurance premiums generally have a high-income [26]. We found higher rates of adherence in high-income patients than in low-income patients. Studies on the impact of economic factors on survival outcomes in CML are scarce. A previous report from Sweden demonstrated that al- though socioeconomic conditions influenced treatment choice, these were not associated with CML survival [27]. However, the Swedish study included not only economic but also social status (i.e., child support and study allowances).

Given that most CML patients are asymptomatic and require long-term treatment to prevent disease progression and achieve optimal response, ensuring 100\% treatment adherence may be challenging. Factors such as older age, living alone, male sex, experiencing medication side effects, a longer time interval between diagnosis and treatment, a longer duration of treatment, poor side effect management, and a high number of concomitant medications are associated with low TKI adherence $[8,11]$. In the present study, the median MPR of the younger ( $<70$ years) and male patients were significantly higher than that of the other age groups and female patients. This finding is in contrast to that of previous studies [11]. However, the median MPRs ( $\geq$ 0.95 ) in both sexes indicated high adherence, and thus, we could not consider sex as a predictor of adherence.

Most studies on CML enrolled patients treated only with imatinib mainly because of insurance coverage $[11,19,22,28]$, and insurance coverage is important in treatment adherence. For example, a previous study from Brazil, where universal health care coverage is challenging, showed that patients had much lower adherence because of a lack of access to drugs [28]. Given that all CML patients in Korea have pharmacy medication coverage, we could better study the association between adherence and outcomes without confounding effects of insurance problems. Interestingly, among the three TKIs, adherence was the lowest to dasatinib compared to that to imatinib or nilotinib, which involves more pills per day and more complicated dosage methods. The median MPR of patients who maintained frontline TKI was higher than that of other patients. This result is expected because maintaining frontline TKI indicates that patients have achieved optimal response or have not experienced uncontrolled adverse events during first-line TKI treatment.

This study had some limitations. First, we had to depend on claims data because of the retrospective nature of the study, and thus, we might have missed some important factors or the data might be unevenly distributed. Second, this study did not consider the CML phase 
during patient inclusion. Third, laboratory results were not available, and therefore, the initial CML risk scores or treatment response (including disease progression) on polymerase chain or cytogenetic testing assessments could not be assessed. Future prospective studies on TKI adherence in CML should assess these factors.

In conclusion, adherence to TKI treatment is an important factor predicting survival outcomes in CML patients. Adherence was better in patients older than 70 years, with higher income and maintenance of frontline TKI. Besides TKI adherence, male sex, young age, and good economic status were associated with better survival outcomes in Korean CML patients.

\section{KEY MESSAGE}

1. Adherence to tyrosine kinase inhibitor (TKI) treatment influences survival outcomes in chronic myeloid leukemia.

2. Age $\leq 70$ years, high income, and maintenance of frontline TKI results in better TKI adherence.

3. Good economic status promotes TKI adherence.

\section{Conflict of interest}

No potential conflict of interest relevant to this article was reported.

\section{Acknowledgments}

This research was supported by the chronic myeloid leukemia working party of The Korean Society of Hematology (CMLWP2018-02). The funders had no role in study design, data collection, and analyses, decision to publish, or manuscript preparation.

\section{REFERENCES}

1. Hehlmann R. Chronic myeloid leukemia in 2020. Hemasphere 2020;4:e468.

2. Carlesso N, Frank DA, Griffin JD. Tyrosyl phosphorylation and DNA binding activity of signal transducers and activators of transcription (STAT) proteins in hematopoietic cell lines transformed by Bcr/Abl. J Exp Med 1996;183:811-
820.

3. Sawyers CL, Callahan W, Witte ON. Dominant negative MYC blocks transformation by ABL oncogenes. Cell 1992;70:901-910.

4. Huang X, Cortes J, Kantarjian H. Estimations of the increasing prevalence and plateau prevalence of chronic myeloid leukemia in the era of tyrosine kinase inhibitor therapy. Cancer 2012;118:3123-3127.

5. Hochhaus A, Larson RA, Guilhot F, et al. Long-term outcomes of imatinib treatment for chronic myeloid leukemia. N Engl J Med 2017;376:917-927.

6. Hochhaus A, Baccarani M, Silver RT, et al. European LeukemiaNet 2020 recommendations for treating chronic myeloid leukemia. Leukemia 2020;34:966-984.

7. Cortes JE, Saglio G, Kantarjian HM, et al. Final 5-year study results of DASISION: the dasatinib versus imatinib study in treatment-naïve chronic myeloid leukemia patients trial. J Clin Oncol 2016;34:2333-2340.

8. Marin D, Bazeos A, Mahon FX, et al. Adherence is the critical factor for achieving molecular responses in patients with chronic myeloid leukemia who achieve complete cytogenetic responses on imatinib. J Clin Oncol 2010;28:2381-2388.

9. Ganesan P, Sagar TG, Dubashi B, et al. Nonadherence to imatinib adversely affects event free survival in chronic phase chronic myeloid leukemia. Am J Hematol 2011;86:471-474.

10. Osterberg L, Blaschke T. Adherence to medication. N Engl J Med 2005;353:487-497.

11. Noens L, van Lierde MA, De Bock R, et al. Prevalence, determinants, and outcomes of nonadherence to imatinib therapy in patients with chronic myeloid leukemia: the ADAGIO study. Blood 2009;113:5401-5411.

12. National Health Insurance Service (KR). History of the NHIS [Internet]. Wonju (KR): National Health Insurance Service, c2010 [cited 2021 Oct 20]. Available from: http:// www.nhis.or.kr/static/html/wbd/g/a/wbdgao203.html.

13. National Health Insurance Service. The Effect of National Health Examination Program on the Early Diagnosis of Diseases, Medical Utilization, and Health Outcome. Seoul (KR): National Health Insurance Service, 2011.

14. Fritz A, Percy C, Jack A, et al. International Classification of Diseases for Oncology. 3rd ed. Geneva (CH): World Health Organization, 2000.

15. Peterson AM, Nau DP, Cramer JA, Benner J, Gwadry-Sridhar F, Nichol M. A checklist for medication compliance 
and persistence studies using retrospective databases. Value Health 2007;10:3-12.

16. Noens L, Hensen M, Kucmin-Bemelmans I, Lofgren C, Gilloteau I, Vrijens B. Measurement of adherence to BCR-ABL inhibitor therapy in chronic myeloid leukemia: current situation and future challenges. Haematologica 2014;99:437-447.

17. Wu EQ, Johnson S, Beaulieu N, et al. Healthcare resource utilization and costs associated with non-adherence to imatinib treatment in chronic myeloid leukemia patients. Curr Med Res Opin 2010;26:61-69.

18. Yood MU, Oliveria SA, Cziraky M, Hirji I, Hamdan M, Davis $\mathrm{C}$. Adherence to treatment with second-line therapies, dasatinib and nilotinib, in patients with chronic myeloid leukemia. Curr Med Res Opin 2012;28:213-219.

19. Kiguchi T, Tauchi T, Ito Y, Miyazawa K, Kimura Y, Ohyashiki K. Compliance with taking imatinib mesylate in patients with chronic myeloid leukemia in the chronic phase. Leuk Res 2009;33:506-508.

20. Wu EQ, Guerin A, Yu AP, Bollu VK, Guo A, Griffin JD. Retrospective real-world comparison of medical visits, costs, and adherence between nilotinib and dasatinib in chronic myeloid leukemia. Curr Med Res Opin 2010;26:28612869.

21. Halpern R, Becker L, Iqbal SU, Kazis LE, Macarios D, Badamgarav E. The association of adherence to osteoporosis therapies with fracture, all-cause medical costs, and all-cause hospitalizations: a retrospective claims analysis of female health plan enrollees with osteoporosis. J
Manag Care Pharm 2011;17:25-39.

22. Darkow T, Henk HJ, Thomas SK, et al. Treatment interruptions and non-adherence with imatinib and associated healthcare costs: a retrospective analysis among managed care patients with chronic myelogenous leukaemia. Pharmacoeconomics 2007;25:481-496.

23. Jabbour E, Saglio G, Radich J, Kantarjian H. Adherence to BCR-ABL inhibitors: issues for CML therapy. Clin Lymphoma Myeloma Leuk 2012;12:223-229.

24. Ector GICG, Visser O, Westerweel PE, Janssen JJWM, Blijlevens NMA, Dinmohamed AG. Primary therapy and relative survival among elderly patients with chronic myeloid leukemia: a population-based study in the Netherlands, 1989-2017. Leukemia 2020;34:3408-3412.

25. de Almeida MH, Pagnano KB, Vigorito AC, Lorand-Metze I, de Souza CA. Adherence to tyrosine kinase inhibitor therapy for chronic myeloid leukemia: a Brazilian single-center cohort. Acta Haematol 2013;130:16-22.

26. Kim YI. National Health Insurance System of Korea. Wonju (KR): National Health Insurance Service, 2015.

27. Larfors G, Sandin F, Richter J, et al. The impact of socio-economic factors on treatment choice and mortality in chronic myeloid leukaemia. Eur J Haematol 2017;98:398-406.

28. Goldberg SL, Chen L, Guerin A, et al. Association between molecular monitoring and long-term outcomes in chronic myelogenous leukemia patients treated with first line imatinib. Curr Med Res Opin 2013;29:1075-1082. 